\title{
Association of Neonatal Myasthenia Gravis with Antibodies against the Fetal Acetylcholine Receptor
}

\author{
Beatrice Vernet-der Garabedian, Miriam Lacokova, * Bruno Eymard, ${ }^{*}$ Evelyne Morel, Michal Faltin, * Jozef Zajac, * \\ Oliver Sadovsky, ${ }^{5}$ Marc Dommergues," Philippe Tripon,' and Jean-François Bach \\ INSERM U 25, Hôpital Necker, Paris, France; *NsP Staré Mesto, Bratislava, Slovensko; ${ }^{\ddagger}$ Hôpital de la Salpétrière, \\ Service de Neurologie, Paris, France; ${ }^{8}$ NsP akademika Dérera, Bratislava, Slovensko; "Maternité de Port Royal, Paris, \\ France; and 'Hôpital Begin, Saint Mande, France
}

\begin{abstract}
The specificities of autoantibodies directed against the acetylcholine receptor ( $A C h R$ ) for embryonic and adult muscle AChR were studied in 22 mothers with myasthenia gravis (MG) and in their newborns using human fetus and normal adult muscle AChR preparations. 12 mothers had transmitted MG to their neonates with, in three cases, antenatal injury. A clear correlation was found between occurrence of neonatal MG (NMG) and the high overall level of antiAChR antibodies (embryonic or adult muscle AChR). However, a strong correlation was also found between occurrence of NMG and the ratio of anti-embryonic AChR to anti-adult muscle (Te/Ta) AChR antibodies $(P<0.0002)$. Taken together, these data suggest that autoantibodies directed against the embryonic form of the $A C h R$ could play a predominant role in the pathogenesis of NMG. Paradoxically, the three cases with antenatal injury presumably the most severe form of NMG, were not associated with high Te/Ta. At the clinical level, these observations could prove helpful in the prediction of transmission of NMG. (J. Clin. Invest. 1994. 94:555-559.) Key words: autoantibody • antigen $\cdot$ autoimmunity $\bullet$ pathogenesis $\bullet$ pregnancy
\end{abstract}

\section{Introduction}

Transient neonatal myasthenia gravis (NMG) ${ }^{1}$ occurs in $~ 20 \%$ of infants born to mothers with myasthenia gravis (MG). Symptoms usually appear within hours after birth and disappear after 2-3 wk except in rare cases with prolonged duration of symptoms. The severity of NMG is highly variable, ranging from mild hypotonia to respiratory distress requiring assisted mechanical ventilation. Antenatal onset leading to multiple joint

This paper was presented in part in abstract form at the 5th National Congress on Neuromuscular diseases of the Association Française contre les myopathies, Strasbourg, France, June 21-25, 1993.

Address all correspondence to Dr. Vernet-der Garabedian, Immunologie clinique, Hôpital Necker, 161 Rue de Sèvres, 75743 Paris CEDEX 15, France.

Received for publication 19 November 1993 and in revised form 22 February 1994.

1. Abbreviations used in this paper: $\mathrm{AChR}$, acetylcholine receptor; $\mathrm{MG}$, myasthenia gravis; NMG, neonatal MG; Ta, muscle AChR antibody titer; Te, anti-embryonic AChR antibody titer.

J. Clin. Invest.

(c) The American Society for Clinical Investigation, Inc.

0021-9738/94/08/0555/05 \$2.00

Volume 94, August 1994, 555-559 contractures, hydramnios, and decreased fetal movements is rare. The disease severity is not correlated to the clinical status of the mother $(1,2)$.

The occurrence of NMG is assumed to be related to the transplacental transfer of maternal antibodies directed against the nicotinic acetylcholine receptor $(\mathrm{AChR})$. The pathogenic role of anti-AChR antibodies in the pathogenesis of MG is supported by several lines of evidence. (a) The transmission of a myasthenic syndrome in animals after passive transfer either of anti-AChR antibodies in MG patient sera or of antiAChR monoclonal antibodies; or $(b)$ The induction of experimental MG after immunization against AChR. However, it is not yet clear why some newborns develop NMG while others remain asymptomatic, despite the presence of serum anti-AChR antibodies (2-8). As observed in our previous study including 52 babies ( 24 presenting NMG), the onset of NMG is clearly correlated to high levels of maternal anti-AChR antibodies (2, $8)$, confirming earlier reports $(3,4)$ but contradicting other studies (5-6). At any rate, there are exceptions to the correlation, with unaffected babies showing high titers of AChR antibodies. In addition there was no clear correlation between the titers of various subsets of anti-AChR antibodies (blocking or modulating) and the clinical status of neonates $(8,9)$.

The nicotinic $\mathrm{AChR}$ is a pentameric glycoprotein complex composed of four different membrane-spanning subunits in a stoichiometry of $\alpha 2 \beta(\gamma$ or $\epsilon) \delta(10,11)$. Two forms of AChR are found in mammalian skeletal muscle cells. The mature form is predominant in innervated adult muscle and the embryonic form is present in fetal and denervated muscle. Embryonic and mature AChR differ by the replacement of the $\gamma$ subunit by its isoform, the $\epsilon$ subunit specifying the mature AChR subtype (12-14). It has been demonstrated using rat and mouse AChR that sera from MG patients react more strongly with the receptors of fetus and denervated muscle than with those of normal adult muscle (15-18). However, the binding of MG sera to rat or mouse $\mathrm{AChR}$ is generally poor because of the high species specificity of MG antibodies (18), and limited data have been obtained using human fetal AChR preparations (19). No study of NMG sera using embryonic AChR has been reported so far except one from our own laboratory using rat AChR (8).

The aim of this study was to investigate the reactivity of sera from myasthenic mothers and from their neonates with both embryonic and adult AChR looking for a possible correlation between antibody specificity and the presence and severity of NMG.

\section{Methods}

Patient sera. 46 sera from mothers with MG and their newborns were obtained from blood collected at delivery and from the umbilical cord at birth or from peripheral veins shortly thereafter. Samples were frozen 
Table I. Clinical Data of Mothers and Their Newborns

\begin{tabular}{|c|c|c|c|c|c|c|}
\hline Case No. & Severity grade* & Thymectomy ${ }^{\ddagger}$ & Age at onset & Duration of disease & $\mathrm{NMG}^{3}$ & Duration of NMG \\
\hline & & & $y r$ & $y r$ & & \\
\hline 1 & IIA & $\mathrm{X}$ & 22 & 1 & 3 & $\mathbf{X}$ \\
\hline 2 & IIA & $\mathrm{T}$ & 27 & 5 & 2 & $10 \mathrm{~d}$ \\
\hline 3 & IIA & $\mathrm{X}$ & 24 & 2 & 2 & $5 \mathrm{~d}$ \\
\hline 4 & In remission & $\mathrm{T}$ & 20 & 8 & $1(2)$ & $2 \mathrm{~d}$ \\
\hline 5 & IIA & $\mathbf{X}$ & $\mathrm{X}$ & $\mathbf{X}$ & 4 & $1 \mathrm{mo}$ \\
\hline 6 & IIA & $\mathbf{T}$ & 12 & 11 & 3 & 5 wk \\
\hline 7 & IIA & $\mathbf{T}$ & 24 & 1 & 4 & $21 \mathrm{~d}$ \\
\hline 8 & IIB & 0 & 22 & 4 & 2 & $>1 \mathrm{mo}$ \\
\hline 9 & IIA & 0 & 35 & 2 & $2(3)$ & $5 \mathrm{~d}$ \\
\hline 10 & IIA & $\mathbf{X}$ & 16 & 19 & $4+\mathrm{AI}$ & $1 \mathrm{yr}$ \\
\hline 11 & Asymptomatic & 0 & $?$ & $?$ & $4+\mathrm{AI}$ & $35 \mathrm{~d}$ \\
\hline 12 & IIB & $\mathbf{X}$ & 12 & 9 & $3(\mathrm{AI}) ?$ & $14 \mathrm{~d}$ \\
\hline 13 & IIB & 0 & 15 & 11 & 0 & 0 \\
\hline 14 & IIB & 0 & 26 & 1 & 0 & 0 \\
\hline 15 & IIA & $\mathrm{T}$ & 25 & 7 & 0 & 0 \\
\hline $16^{\| \prime}$ & IIB & $\mathrm{T}$ & 23 & $2(4)$ & $0-0$ & $0-0$ \\
\hline 17 & IIA & $\mathbf{T}$ & 19 & 14 & 0 & 0 \\
\hline 18 & In remission & $\mathrm{T}$ & 16 & 19 & 0 & 0 \\
\hline 19 & IIA & $\mathbf{X}$ & $\mathbf{x}$ & $\mathbf{X}$ & 0 & 0 \\
\hline 20 & IIA & 0 & 21 & 3 & 0 & 0 \\
\hline 21 & IIA & $\mathbf{X}$ & 18 & 10 & 0 & 0 \\
\hline 22 & I & 0 & 38 & 1 & 0 & 0 \\
\hline 23 & IIB & $\mathrm{T}$ & 22 & 2 & $0 ?$ & 0 \\
\hline
\end{tabular}

* Grading according to Osserman's classification.

₹ Thymectomy: $T$, yes; 0 , no; $X$, unknown.

${ }^{8}$ Severity of tNMG: 0 , no NMG; 1 , slight hypotonia; 2 , severe hypotonia; 3 , suction problems; 4 , assisted ventilation; $A I$, antenatal injury.

"Mother with two newborns.

and stored at $-70^{\circ} \mathrm{C}$. The diagnosis of MG and NMG was based on clinical, pharmacological, electromyographical, and immunological (anti-AChR antibodies) criteria, as previously reported (2). Table I summarizes clinical data of the 22 mothers and their 23 newborns studied (one mother had 2 babies). 12 mothers (Nos. 1 to 12) transmitted MG to their newborns (hereafter called "transmitting mothers"). Of the 12 affected newborns, 10 presented common NMG, i.e., a transient myasthenic syndrome without fetal involvement, and two presented NMG with antenatal injury. Case No. 12 was included in this group although fetal involvement was not reported because this child, born at 39 wk by caesarian section, had potential fetal damage with an Apgar score of 4 in the first minute and neurological disturbances (global hypotonia and troubles of suction). 10 mothers (Nos. 13 to 22) had 11 unaffected newborns (hereafter called "nontransmitting mothers").

AChR preparations. Adult human muscle was obtained from a limb of an otherwise healthy individual (without muscle ischemia or denervation) after a traffic accident. Human fetuses (8-14 wk) were obtained from voluntary pregnancy terminations. They were stored at $-70^{\circ} \mathrm{C}$ before use. AChR was extracted using Triton X-100 as previously described (9). The antigen from adult muscle had quantitative and qualitative immunoreactivity properties similar to antigen from normal adult muscles previously described $(2,9)$.

Antibody assay. Anti-AChR antibody titers were determined by a radioimmunoprecipitation assay using solubilized $\mathrm{AChR}$ from adult or fetal skeletal muscle ( $0.25 \mathrm{nM}$ final concentration) and complexed to ${ }^{125} \mathrm{I}-\alpha$-Bgt as previously described (9). Results were expressed as nmol bound/I serum.

Statistical analysis. Mann-Whitney's $U$-test and Spearman's rank correlation test were used for statistical analysis.

\section{Results}

Comparison of reactivity of $M G$ sera with adult and embryonic $A C h R$. We evaluated the serum anti-AChR antibody titers of MG mothers and of their newborns with the embryo and adult muscle antigen preparations. Titers varied over a broad range whatever antigen was used, from 2.7 to $2,703 \mathrm{nM}$ with embryonic AChR and from 1 to $332 \mathrm{nM}$ with adult AChR. As shown on Fig. 1, there was a good correlation between antibody titers detected in the two assays, with a clear tendency for higher titers using the embryonic receptor, i.e., a 4.5-fold average difference $(P<0.0001)$. Newborns generally had the same pattern of antibody reactivity towards the two forms of receptor as their mothers, but with lower titers (Fig. 2, $a-d$ ). In 5 out of 21 cases, anti-AChR antibody titers were higher in neonates than in their mothers. These sera contained high IgG levels. Of these five samples, three were from umbilical blood, known to have higher IgG levels than peripheral blood (2).

Correlation with NMG. Anti-AChR antibody titers were higher in "transmitting" than in "nontransmitting mothers" (Fig. 2, $a$ and $c$ ) and correspondingly higher in affected than in asymptomatic newborns (Fig. $2, b$ and $d)(P<0.003)$. This difference was found with both embryonic and adult muscle AChR. It was statistically significant both for maternal sera ( $P$ $<0.002$ and $P<0.01$, respectively) and for newborn sera ( $P$ $<0.002$ and $P<0.003$, respectively). 


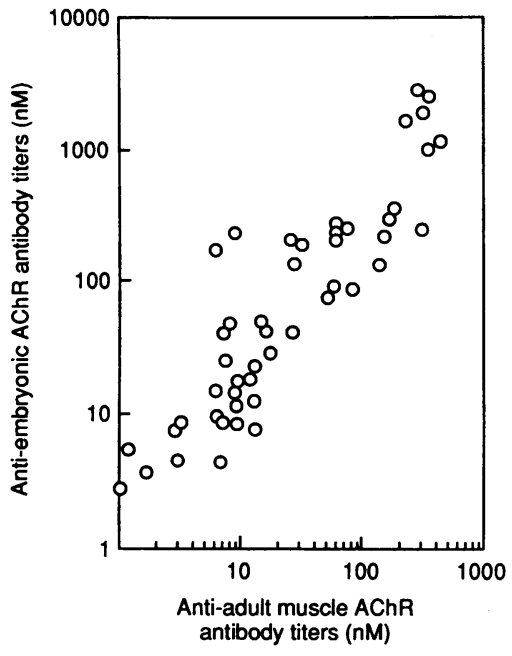

Considering the overall higher level of both anti-embryonic and anti-adult AChR antibodies in "transmitting" than in "nontransmitting mothers," the possibility of a preferential role of anti-embryonic AChR antibodies was studied in terms of antiembryonic AChR/anti-adult muscle AChR antibody titer ratio ( $\mathrm{Te} / \mathrm{Ta}$ ) for individual sera rather than in absolute values. Since
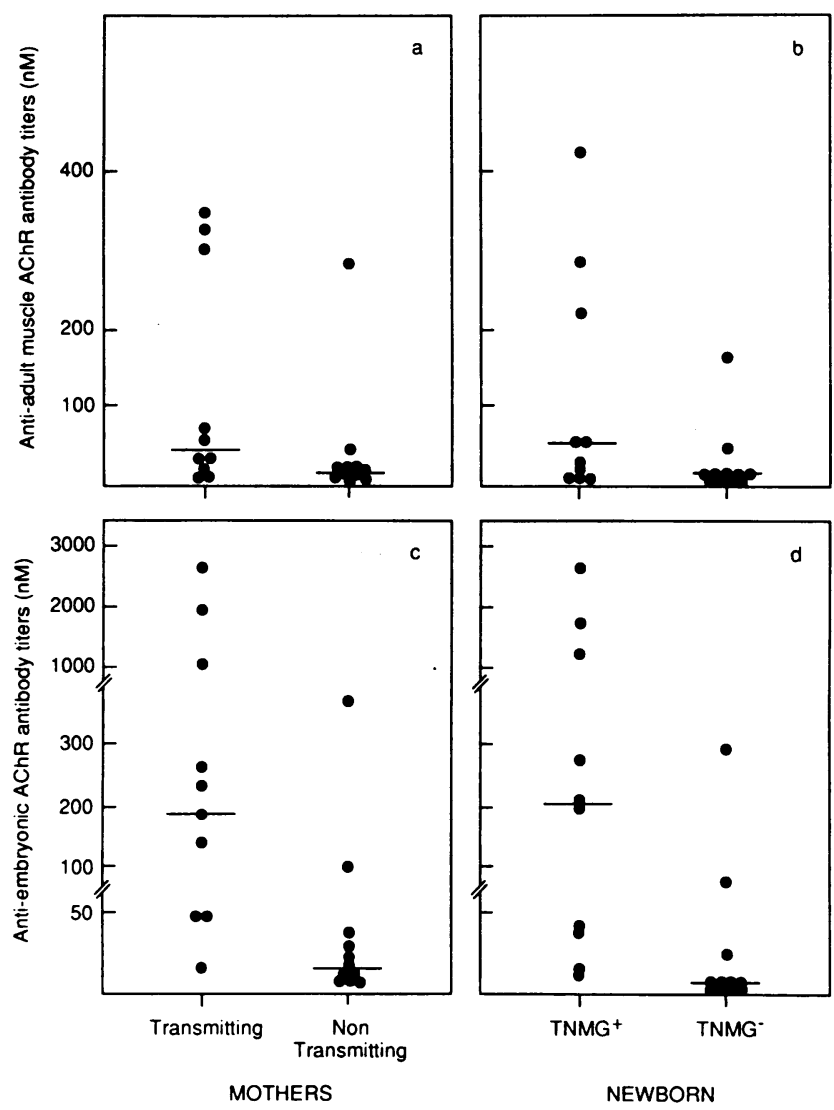

Figure 2. Distribution of anti-adult muscle ( $a$ and $b$ ) and anti-embryonic ( $c$ and $d$ ) AChR antibody titers in "transmitting" and "nontransmitting mothers" ( $a$ and $c$ ) and in their affected (NMG+) and asymptomatic (NMG-) newborns ( $b$ and $d$ ). Median values are indicated for each group of patients.

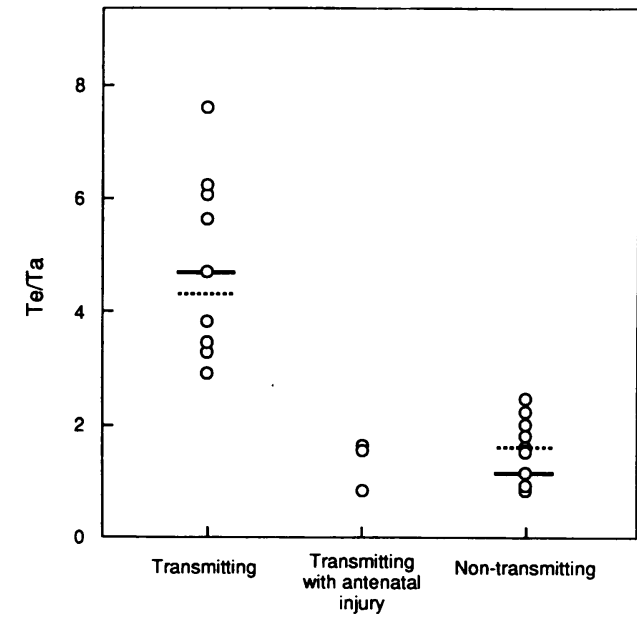

Figure 3. Relationship between NMG transmission and levels of antiembryonic to anti-muscle AChR antibody titer ratio ( $\mathrm{Te} / \mathrm{Ta}$ ) of MG mothers' sera. Median values $(-)$ and means $(\cdots)$ ) are indicated for each group of patients.

antibody titers of maternal and newborn sera were closely related, the complete study was performed on the maternal sera. This ratio had an average value of $2.8(\mathrm{SD}=1.9)$ in myasthenic mothers taken as a whole group.

As shown on Fig. 3, sera of "common-NMG-transmitting mothers" showed higher Te/Ta than sera from "nontransmitting mothers."' Mean Te/Ta ratios were 4.86 (ranging from 2.91 to 7.61 ) for "transmitting mothers" versus 1.60 (from 0.82 to 2.45 ) for "nontransmitting mothers." This difference is highly statistically significant $(P<0.0002)$. Paradoxically sera from the three mothers having transmitted NMG with antenatal injury tended to present a lower $\mathrm{Te} / \mathrm{Ta}$ ratio than other "transmitting mothers."

No correlation was observed between either antibody titers or $\mathrm{Te} / \mathrm{Ta}$ ratio with the severity of the disease in the mothers or in their affected children.

Combining the two criteria ( $\mathrm{Te} / \mathrm{Ta}$ ratio, anti-adult muscle AChR antibody titers), a good correlation is observed between high $\mathrm{Te} / \mathrm{Ta}$ ratio, high anti-adult muscle $\mathrm{AChR}$ antibody titers and NMG (Fig. 4). Seven out of the nine sera of "common NMG-transmitting mothers" showed a Te/Ta ratio $>3$ and anti-adult muscle AChR titer $>20 \mathrm{nM}$, versus none of the 10 sera of "nontransmitting mothers."

\section{Discussion}

This study shows that sera from myasthenic mothers transmitting the disease to their newborns presenting anti-AChR antibodies differ from sera from "nontransmitting mothers" both quantitatively (anti-AChR titers) and qualitatively ( Te/Ta ratio). It appears that NMG induction is usually associated with particularly high titers of anti-human embryonic AChR antibodies. This finding had not emerged from our previous studies using rat AChR, probably due to the high level of species specificity of anti-AChR autoantibodies $(8,18)$. It is interesting to note that several studies have shown, independently of NMG transmission, that MG sera react more strongly with rat or mouse embryonic than adult muscle $\operatorname{AChR}(15,20,21)$.

The relevance of these data to the pathogenesis of NMG is 


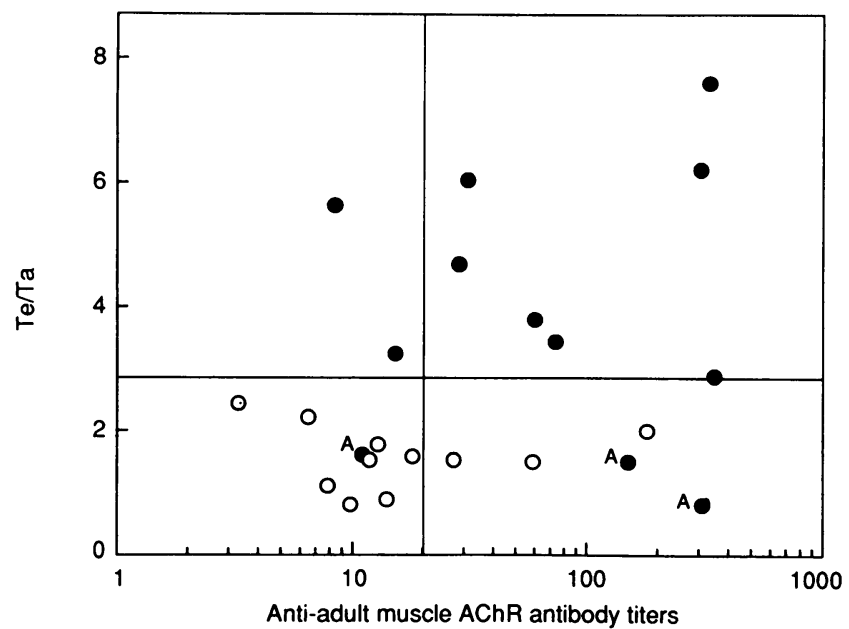

Figure 4. Relationship between anti-adult muscle AChR antibody levels of "transmitting" $(\bullet)$, "transmitting" with antenatal injury $(A \bullet)$ and "nontransmitting" $(O)$ mothers and ratios of anti-embryonic to antiadult muscle AChR antibody titers.

difficult to establish in view of the limited data presently available on the maturation of human AChR. One should note, however, that in the study reported by Hesselmans et al. (22), fetal AChR was no longer detected in 33-wk-old fetuses. If these data are confirmed, they would suggest that NMG is not due to a particular effect of anti-embryonic AChR antibodies on embryonic AChR persisting in newborns. They would rather indicate that anti-embryonic AChR antibodies induce alterations of fetal maturation of the AChR. This maturation would be delayed in NMG, which could explain abnormal late (postnatal) sensitivity to anti-embryonic AChR antibodies. This mechanism is reminiscent of current hypotheses on the effect of maternal anti-thyroid autoantibodies on thyroid primordium maturation and migration leading to congenital hypothyroidism with common ectopy (23).

However, this interpretation should be taken with caution since the maturation kinetics of AChR reported in Hesselmans's study is at variance with those reporting other mammalian species ( rat and calf) in which changes from fetal to adult receptor type progressively occur in the first weeks after birth $(12,14$, $20,24)$. If the same maturation kinetics occurred in man, the hypothesis of a preferential role of anti-embryonic AChR antibodies on the embryonic neonate AChR appears to be most likely. The case of the three presumably severe NMG cases associated with low $\mathrm{Te} / \mathrm{Ta}$ ratios is difficult to explain with the reservation of the low number of serum samples studied in this category. One may postulate that the nature of NMG with antenatal injury is due to another yet unknown mechanism.

With regard to fine antigenic specificity of anti-AChR antibodies, the difference between anti-embryonic and anti-adult muscle AChR titers in sera from transmitting mothers may be related to the presence of one or more epitopes specific for embryonic AChR or of a partly common epitope shared on the two AChR forms. These epitopes are probably located on the $\gamma$ subunit and its $\epsilon$ isoform rather than on the $\alpha$ subunit (so far identical in embryonic and mature AChRs) although the latter is generally considered as the main target of $\mathrm{MG}$ anti-AChR antibodies and contains the main immunogenic region (25). Localization of the epitope on an $\alpha-\gamma$ conformation dependent structure is also conceivable. Whether distinct antibody subpopulations specifically react with the two AChR forms or whether crossreacting antibodies recognize both embryonic and adult muscle AChRs with different affinities remains to be determined.

In conclusion, this study provides an interesting example of autoantibodies showing a particular pathogenicity in fetuses or newborns. Other antibodies of this type have already been described such as the anti-Ro (SS-A) autoantibodies which can induce atrio-ventricular heart block (eventually leading to lethal heart arrest) of the fetus without affecting the mother's heart (26). Similarly, as mentioned above, it has been postulated that anti-thyroid autoantibodies could favor abnormal migration of the thyroid primordium since congenital hypothyroidism is significantly associated with maternal anti-thyroid autoimmunity (23).

These results should prompt further studies on the maturation of human AChR and its functional expression. At the clinical level, they offer interesting possibilities for predicting the occurrence of NMG in fetus and newborns in pregnant myasthenic patients.

\section{Acknowledgments}

We greatly thank Mrs. A. Perdereau and Mr. F. Bustarret for helpful technical assistance and Mrs. L. Gomez for assistance in preparation of the manuscript. Our grateful thanks to Dr. C. R. Gardner for helpful discussion during the writing of this paper. Our thanks to Dr. V. Prochazka for providing material. We are indebted to Dr. M. Trnkova and Dr. S. Krcméry for assistance with the availability of samples. We are grateful to Prof. S. Cagan and Dr. J. Hromec for their support throughout to this study.

This work was supported in part by the Association Française contre les Myopathies.

\section{References}

1. Papazian, O. 1992. Transient neonatal myasthenia gravis. J. Child Neurol. 7:135-141

2. Morel, E., B. Eymard, B. Vernet-der Garabedian, C. Pannier, O. Dulac, and J. F. Bach. 1988. Neonatal myasthenia gravis: a new clinical and immunologic appraisal on 30 cases. Neurology. 38:138-142.

3. Keesey, K., J. Lindstrom, H. Cokely, and C. Hermann. 1977. Anti-acetylcholine receptor antibody in neonatal myasthenia gravis. $N$. Engl. J. Med. 296:55.

4. Ohta, M., F. Matsubara, K. Hayashi, K. Nakao, and H. Nishitani. 1981. Acetylcholine receptor antibodies in infants of mothers with myasthenia gravis. Neurology. 31:1019-1022.

5. Lefvert, A. K., and P. O. Osterman. 1983. Newborn infants to myasthenic mothers: a clinical study and an investigation of acetylcholine receptor antibodies in 17 children. Neurology. 33:133-138.

6. Bartoccioni, E., A. Evoli, C. Casali, C. Scoppetta, P. Tonali, and C. Provenzano. 1986. Neonatal myasthenia gravis: clinical and immunological study of seven mothers and their newborn infants. J. Neuroimmunol. 12:155-161.

7. Tzartos, S. J., A. Efthimiadis, E. Morel, B. Eymard, and J. F. Bach. 1990. Neonatal myasthenia gravis: antigenic specificities of antibodies in sera from mothers and their infants. Clin. Exp. Immunol. 80:376-380.

8. Eymard, B., B. Vernet-der Garabedian, S. Berrih-Aknin, C. Pannier, Bach J. F., and E. Morel. 1991. Anti-acetylcholine receptor antibodies in neonatal myasthenia gravis: heterogeneity and pathogenic significance. J. Autoimmun. 4:185-195.

9. Vernet-der Garabedian, B., B. Eymard, J. F. Bach, and E. Morel. 1989. Alpha-bungarotoxin blocking antibodies in neonatal myasthenia gravis: frequency and selectivity. J. Neuroimmunol. 21:41-47.

10. Galzi, J. L., F. Revah, A. Bessis, and J. P. Changeux. 1991. Functional architecture of the nicotinic acetylcholine receptor: from electric organ to brain. Annu. Rev. Pharmacol. Toxicol. 31:37-72.

11. Karlin, A. 1991. Explorations of the nicotinic acetylcholine receptor Harvey Lect. 85:71-107.

12. Mishina, M., T. Takai, K. Imoto, M. Noda, T. Takahashi, S. Numa, C. 
Methfessel, and B. Sakmann. 1986. Molecular distinction between fetal and adult forms of muscle acetylcholine receptor. Nature (Lond.). 321:406-411.

13. Witzemann, V., B. Barg, M. Criado, E. Stein, and B. Sakmann. 1989. Developmental regulation of five specific mRNAs encoding acetylcholine recepto subtypes in rat muscle. FEBS (Fed. Eur. Biochem. Soc.) Lett. 242:419-424.

14. Brenner, H. R., V. Witzemann, and B. Sakmann. 1990. Imprinting of acetylcholine receptor messenger RNA accumulation in mammalian neuromuscular synapses. Nature (Lond.). 344:544-547.

15. Weinberg, C. B., and Z. W. Hall. 1979. Antibodies from patients with myasthenia gravis recognize determinants unique to extrajunctional acetylcholine receptors. Proc. Natl. Acad. Sci. USA 76:504-508.

16. Dwyer, S. D., J. Bradley, R. L. Furner, and G. E. Kemp. 1981. Immunochemical properties of junctional and extrajunctional acetylcholine receptor. Brain Res. 217:23-40.

17. Souroujon, M. C., S. Pizzighella, D. Mochly-Rosen, and S. Fuchs. 1985 Antigenic specificity of acetylcholine receptor in developing muscle. Studies with monoclonal antibodies. J. Neuroimmunol. 8:159-166.

18. Lennon, V. A., and G. E. Griesmann. 1989. Evidence against acetylcholine receptor having a main immunogenic region as target for autoantibodies in myasthenia gravis. Neurology. 39:1069-1076.

19. Lotwick, H., R. Harrison, G. Lunt, and P. Behan. 1983. Interaction of fetal and adult human acetylcholine receptors with serum from patients with myasthenia gravis. J. Neuroimmunol. 4:167-174.
20. O'malley, J. P., S. J. Wilson, and J. J. Bray. 1988. Loss of antigenic properties of acetylcholine receptors in rat skeletal muscle after birth. J. Neuroimmunol. 18:187-196.

21. Silberstein, L., P. D. Gorin, and Z. W. Hall. 1984. Differentiation of the acetylcholine receptor at developing neuromuscular junctions. In Neuromuscular Diseases. G. Serratrice editor. Raven Press, New York. 9-14.

22. Hesselmans, L. F., F. G. Jennekens, C. J. Van Den Oord, H. Veldman, and A. Vincent. 1989. Immunoreactivity to fetal acetylcholine receptor disappears during the 33d week of human fetal development and reappears with denervation. Second European Conference on Myasthenia Gravis. (Abstr.) Tremezzo (Italy) June 12-14, 1989.

23. Van Der Gaag, R. D., H. A. Drexhage, and J. H. Dussault. 1986. Role of maternal immunoglobulins blocking TSH-induced thyroid growth in sporadic forms of congenital hypothyroidism. Lancet. I:246-250.

24. Hall, Z. W., P. D. Gorin, L. Silberstein, and C. Bennett. 1985. A postnatal change in the immunological properties of the acetylcholine receptors at rat muscle endplates. J. Neurosci. 5:730-734.

25. Tzartos, S. J., M. E. Seybold, and J. Lindstrom. 1981. Specificities of antibodies to acetylcholine receptors in sera from myasthenia gravis patients measured by monoclonal antibodies. Proc. Natl. Acad. Sci. USA 79:188-192.

26. Watson, R. M., A. T. Lane, N. Barnett, W. B. Bias, F. C. Arnett, and T. Provost. 1984. Neonatal lupus erythematosus. A clinical, serological and immunogenetic study with review of the literature. Medicine. 63:362-378. 